\title{
COMPACT GENERATION IN PARTIALLY ORDERED SETS
}

\author{
MARCEL ERNÉ
}

(Received 10 August 1984)

Communicated by H. Lausch

\begin{abstract}
Severar "classical" results on algebraic complete lattices extend to algebraic posets and, more generally, to so called compactly generated posets; but, of course, there may arise difficulties in the absence of certain joins or meets. For example, the property of weak atomicity turns out to be valid in all Dedekind complete compactly generated posets, but not in arbitrary algebraic posets. The compactly generated posets are, up to isomorphism, the inductive centralized systems, where a system of sets is called centralized if it contains all point closures. A similar representation theorem holds for algebraic posets; it is known that every algebraic poset is isomorphic to the system $i(Q)$ of all directed lower sets in some poset $Q$; we show that only those posets $P$ which satisfy the ascending chain condition are isomorphic to their own "up-completion" $i(P)$. We also touch upon a few structural aspects such as the formation of direct sums, products and substructures. The note concludes with several applications of a generalized version of the Birkhoff Frink decomposition theorem for algebraic lattices.
\end{abstract}

1980 Mathematics subject classification (Amer. Math. Soc.): 06 A 10, 06 A 23.

Keywords and phrases: poset, up-complete, compactly generated, algebraic, Scott completion, weakly atomic, irreducible element.

\section{Terminology and basic facts}

Given a partially ordered set ("poset") $P$ with order relation $\leqslant$, we denote by $\downarrow y$ the principal ideal generated by $y \in P$, i.e.

$$
\downarrow y:=\{x \in P: x \leqslant y\} \quad(y \in P) .
$$

For $Y \subseteq P$,

$$
\downarrow Y:=\bigcup\{\downarrow y: y \in Y\}
$$

(C) 1987 Australian Mathematical Society $0263-6115 / 87 \$ A 2.00+0.00$ 
denotes the lower set or decreasing set generated by $Y$. The lower sets form a closure system $\theta(P)$ which is also a topology, called the Alexandroff completion of $P$. An Alexandroff discrete topology, or, for short, an $A$-topology, is a system of sets which is closed under arbitrary unions and intersections. It is well known that the assignment $P \mapsto \theta(P)$ yields a one-to-one correspondence between partially ordered sets and $T_{0}-A$-topologies (cf. [1]). A subset $D$ of $P$ is directed if every finite subset of $D$ has an upper bound in $D$ (in particular $D \neq \varnothing$ ). A system $\mathfrak{X}$ of sets is called inductive if for every subsystem $\mathfrak{Y}$ of $\mathfrak{X}$ which is directed by inclusion, the union $\cup \mathfrak{Y}$ also belongs to $\mathfrak{X}$. It suffices to postulate this property for nonempty well-ordered subsystems $\mathfrak{Y}$ (cf. Mayer-Kalkschmidt and Steiner [16]). For any poset $P$, the system $i(P)$ of all directed lower sets ("ideals" in the sense of [10]) is inductive; but in contrast to $\theta(P)$, it is not closed under arbitrary unions of non-empty subsystems unless $P$ is a linearly ordered set.

A poset $P$ is called up-complete if every directed subset $D$ of $P$ has a least upper bound ("join"), denoted by VD. Again, it suffices to postulate the existence of joins for all non-empty well-ordered subsets of $P$ in order to guarantee up-completeness (cf. Cohn [4; 1.5.9]). Of course, every inductive system is an up-complete poset with respect to inclusion $\subseteq$, but a system which is up-complete with respect to inclusion need not be inductive (see Proposition 1). Now the well-known concept of algebraic lattices ("compactly generated lattices" in the sense of Crawley and Dilworth [5]) extends to posets as follows. An element $x$ of an up-complete poset $P$ is called compact if, for every directed subset (or, equivalently, for every non-empty well-ordered subset) $D$ of $P$ with $x \leqslant \vee D$, there exists a $y \in D$ with $x \leqslant y$. Thus $x$ is compact if and only if $x \in \cap\{Y \in$ $i(P): x \leqslant \vee Y$ \}. The subposet of all compact elements of $P$ is denoted by $\kappa(P)$, and $P$ is said to be compactly generated if each element of $P$ is a join of compact elements; moreover, $P$ is called algebraic if for each $y \in P$ there exists a directed set $D$ of compact elements such that $y=V D$. In this case, one may assume without loss of generality that $D$ is the set of all compact elements dominated by $y$. For complete lattices, the properties of being compactly generated and algebraic are equivalent, but this equivalence fails for arbitrary posets, as our first example shows.

EXAMPLE 1. Let $X$ be an uncountable set, and let $S$ denote the system of all subsets of $X$ which have either at most one element or a countable complement. This system is inductive and closed under countable intersections. In particular, it is an up-complete $\wedge$-semilattice with respect to inclusion. The compact elements of $S$ are the singletons and the empty set. Hence $S$ is compactly generated but not algebraic. 
Of course, every poset satisfying the ascending chain condition (a.c.c.) is algebraic (since each element of such a poset is compact). Further examples of algebraic posets which are not complete lattices (except for trivial cases) are the systems of finite character, i.e. systems $\mathfrak{X}$ having the property that a set $Y$ belongs to $X$ if and only if every finite subset of $Y$ belnogs to $X$. Obviously any such system is inductive, and the finite members of $\mathfrak{X}$ are precisely the compact ones. For example, the system of all chains (linearly ordered subsets) of a fixed poset, or the system of all linearly independent subsets of a vector space, partially ordered by inclusion, are algebraic posets. Notice that the only systems of finite character which are complete lattices (with respect to inclusion) are the power sets. A general construction principle for compactly generated (respectively, algebraic) posets will be described in the next paragraph.

\section{Set representation of compactly generated and algebraic posets}

A famous representation theorem due to Birkhoff and Frink [3] states that the algebraic complete lattices are, up to isomorphism, precisely the inductive closure systems. This result can be generalized to compactly generated posets as follows.

By a centralized system on a set $X$ we mean a system $\mathfrak{X}$ of subsets of $X$ with the property that each point closure

$$
C_{x}(\mathfrak{X})=\bigcap\{Y \in \mathfrak{X}: x \in Y\} \quad(x \in X)
$$

belongs to $\mathfrak{X}$. Obviously, every closure system is centralized, but not conversely.

Proposition 1. A centralized system $\mathfrak{X}$ is inductive if and only if it is up-complete with respect to inclusion and the point closures are compact members of $\mathfrak{X}$. In particular, every inductive centralized system is a compactly generated poset.

Proof. If $\mathfrak{X}$ is inductive and centralized, then $\mathfrak{X}$ is up-complete, and the point closures are compact because directed joins agree with the corresponding unions.

Conversely, assume $\mathfrak{X}$ is up-complete and each point closure is compact. Then every directed subsystem $\mathfrak{Y}$ has a join $V \mathfrak{V}$ in $\mathfrak{X}$, and it suffices to prove $\mathrm{V} \mathfrak{Y} \subseteq \mathrm{U} \mathfrak{V}$. But $x \in \mathrm{V} \mathfrak{Y} \in \mathfrak{X}$ implies that $C_{x}(\mathfrak{X}) \subseteq \mathrm{V} \mathfrak{Y}$, and, as $C_{x}(\mathfrak{X})$ is compact, $x \in C_{x}(\mathfrak{X}) \subseteq Y \subseteq \cup \mathfrak{V}$ for some $Y \in \mathfrak{V}$.

A system $\mathfrak{X}$ of sets such that different points have disjoint closures, i.e. such that $C_{x}(\mathfrak{X}) \neq C_{y}(\mathfrak{X})$ for $x \neq y$, will be referred to as a $T_{0}$-system. A standard extension of a poset $P$ is a subsystem of $\theta(P)$ containing all principal ideals of $P$. A straightforward argument shows that a system of sets is a centralized $T_{0}$-system 
if and only if it is a standard extension of a (unique) poset. By a theorem due to B. Banaschewski [2], the standard extensions of a poset $P$ (together with the natural embeddings $x \mapsto \downarrow x)$ form a representative system for the join-dense order embeddings of $P$ (where $f: P \rightarrow Q$ is join-dense if each element of $Q$ is a join of elements in the image of $f$ ). For further background concerning standard extensions, see [9].

Proposition 2. For two posets $P$ and $Q$, the following two statements are equivalent.

(a) $P$ is compactly generated, and $Q$ is isomorphic to $\kappa(P)$.

(b) $P$ is isomorphic to an inductive standard extension of $Q$ whose compact elements are exactly the principal ideals of $Q$.

Proof. (a) $\Rightarrow$ (b). Given an isomorphism $f: Q \rightarrow \kappa(P)$, define an embedding g: $P \rightarrow \theta(Q)$ by $g(y):=f^{-1}[\downarrow y \cap \kappa(P)]$. The image $\mathfrak{X}=g[P]$ is a standard extension of $Q$ since for $x \in Q$, we have $f^{-1}[\downarrow f(x) \cap \kappa(P)]=\downarrow x$. Being an isomorphism between $P$ and $\mathfrak{X}, g$ maps $\kappa(P)$ onto $\kappa(\mathfrak{X})$, and so $\kappa(\mathfrak{X})=$ $\left\{f^{-1}[\downarrow y \cap \kappa(P)]: y \in \kappa(P)\right\}$ is the set of all principal ideals of $Q$ (because $f$ maps $Q$ onto $\kappa(P)$ ). Furthermore, by Proposition 1, $\mathfrak{X}$ must be inductive. (Notice that the point closures of a standard extension are exactly the principal ideals.)

(b) $\Rightarrow$ (a). Let $g: P \rightarrow \mathfrak{X}$ be an isomorphism between $P$ and an inductive standard extension $\mathfrak{X}$ of $Q$ with $\kappa(\mathfrak{X})=\{\downarrow x: x \in Q\}$. Then, by Proposition 1, $\mathfrak{X}$ and $P$ are compactly generated posets. Moreover, the restriction of $g$ to $\kappa(P)$ induces an isomorphism between $\kappa(P)$ and $\kappa(\mathfrak{X})$, and $\kappa(\mathfrak{X})$ is isomorphic to $Q$ via the map $x \mapsto \downarrow x$.

COROLlARY 1. For a poset $P$, the following three conditions are equivalent.

(a) $P$ is compactly generated.

(b) $P$ is isomorphic to an inductive standard extension of some poset $Q$.

(c) $P$ is isomorphic to an inductive centralized system

In (b), one can choose $Q=\kappa(P)$.

Proposition 2 enables us to determine (up to isomorphism) all compactly generated posets with a fixed given poset $Q$ of compact elements: a system of representatives for these posets is the set (!) of all inductive standard extensions of $Q$ whose compact elements are the principal ideals of $Q$. Only one of these representatives is an algebraic poset, namely the "algebraic up-completion" $i(Q)$; and indeed, this is the least inductive standard extension of $Q$, while $\theta(Q)$ is the greatest one. Consequently, an arbitrary compactly generated poset $P$ contains a unique algebraic poset $\tilde{P}$ with $\kappa(\tilde{P})=\kappa(P)$. 
For any up-complete poset $P$, define the Scott operator $\Gamma$ by

$$
\Gamma: \mathfrak{B}(P) \rightarrow \mathfrak{B}(P), \quad Y \mapsto \downarrow\{\bigvee D: D \subseteq \downarrow Y, D \text { directed }\}
$$

This operator is extensive and preserves finite unions, but it is in general not a closure operator. However, the system

$$
\gamma(P)=\{A \subseteq P: \Gamma(A)=A\}
$$

of all Scott-closed sets is a topological closure system contained in $\theta(P)$. The corresponding Scott-open sets form the so-called Scott topology $\sigma(P)$ (cf. [7], [10]). Every algebraic poset is continuous (see [10], [12], [13], [14]). It is an important property of continuous posets that their Scott operator is always a closure operator and that their Scott topology $\sigma(P)$ is completely distributive (see [7], [14], [15]). In order to give a characterization of algebraic posets in terms of their "Scott completion" $\gamma(P)$, we need the notion of prime elements. Call an element $p$ of a complete lattice $L \vee$-prime if for every finite subset $Y$ of $L, p \leqslant \vee Y$ implies that $p \in \downarrow Y$. The set of all $\vee$-primes of $L$ is denoted by $\pi_{\vee}(L)$ and is called the $\vee$-spectrum of $L$. Similarly, we define $V$-primes by dropping the finiteness condition on $Y$ and the $V$-spectrum $\pi \checkmark(L)$. A complete lattice in which every element is a join of $V$-primes will be referred to as an A-lattice, in view of the well-known fact that such lattices are, up to isomorphism, precisely the $A$-topologies. There are various different characterizations of $A$-lattices; for example, they may be identified as the completely distributive algebraic lattices (see also [8]).

Some of the equivalences collected in the subsequent proposition are already known (cf. R.-E. Hoffmann [12], [13], [14]; J. D. Lawson [15]). We list them for the sake of completeness and easy reference.

Proposition 3. For an up-complete poset $P$, the following conditions are equivalent.

(a) $P$ is algebraic.

(b) $\gamma(P)$ is isomorphic to the $A$-topology $\theta(Q)$ of some poset $Q$.

(c) $\gamma(P)$ (respectively $\sigma(P))$ is an A-lattice.

(d) $\gamma(P)$ is algebraic.

(e) $P$ is isomorphic to the $V$-spectrum $\pi_{V}(L)$ of some A-lattice $L$.

(f) $P$ is isomorphic to the up-completion $i(Q)$ of some poset $Q$.

In (b) and (f), one may take $Q=\kappa(P)$, and in (e), $L=\gamma(P)$.

Proof. (a) $\Rightarrow$ (b). The following map is an isomorphism:

$$
f: \gamma(P) \rightarrow \theta(\kappa(P)), \quad A \mapsto A \cap \kappa(P) .
$$

Indeed, the inverse isomorphism is given by

$$
g: \theta(\kappa(P)) \rightarrow \gamma(P), \quad Y \rightarrow \Gamma(Y)
$$


(where $\Gamma$ is the Scott operator of $P$ ). This map is well-defined because the Scott operator of an algebraic poset is a closure operator.

(b) $\Rightarrow$ (c) $\Rightarrow$ (d). These are clear.

(d) $\Rightarrow$ (c). This follows from the fact that a $\Lambda$-distributive algebraic lattice is already an $A$-lattice (see [8]), and $\gamma(P)$ is always $\Lambda$-distributive, being a topological closure system.

(c) $\Rightarrow$ (e). If $\gamma(P)$ is an $A$-lattice, then it is completely distributive, and consequently $P$ is a continuous poset isomorphic to $\pi_{\curlyvee}(\gamma(P)$ ) (cf. [7], [14] and [15]).

(e) $\Rightarrow(f)$. We may assume $P \cong \pi_{\bigvee}(\theta(Q))$ for some poset $Q$. But a direct inspection shows that $\pi \backslash(\theta(Q))=i(Q)$ (cf. [12]).

$(\mathrm{f}) \Rightarrow(\mathrm{a})$. This is clear (see the remark after Corollary 1 ).

COROllary 2. For two posets $P$ and $Q$, the following four conditions are equivalent.

(a) $P$ is algebraic, and $\kappa(P) \cong Q$.

(b) $P$ is up-complete, and $\gamma(P) \cong \theta(Q)$.

(c) $P \cong \pi_{\vee}(L)$ and $Q \cong \pi_{\vee}(L)$ for some $A$-lattice $L$.

(d) $P \cong i(Q)$.

Using these isomorphisms, one can show easily that the following three categories are equivalent.

\begin{tabular}{l|l|l}
\hline & objects & morphisms \\
\hline
\end{tabular}

For more general investigations on such categories, see [9] and [18].

Next let us characterize those posets $P$ which are isomorphic to their own algebraic up-completion $i(P)$. Such a characterization has been established in the case of complete lattices by D. Higgs [11]. However, the proof given in [11] is rather complicated, involves tools of transfinite induction, and requires the completeness hypothesis. The subsequent proof is easier and works in the general setting of posets. 
Proposition 4. For a poset $P$, the following conditions are equivalent.

(a) $P$ is up-complete, and every element of $P$ is compact.

(b) $P$ satisfies the a.c.c.

(c) The map $\eta: P \rightarrow i(P), y \mapsto \downarrow y$ is an isomorphism.

(d) $P$ is isomorphic to $i(P)$.

(e) $P$ is up-complete, and $\gamma(P)=\theta(P)$.

Proof. It is well known that $P$ satisfies the a.c.c. if and only if each directed subset of $P$ has a greatest element. From this observation the implications (a) $\Rightarrow$ (b) $\Rightarrow$ (c) $\Rightarrow$ (d) and the equivalence (b) $\Leftrightarrow$ (e) are evident.

We prove the remaining implication (d) $\Rightarrow$ (a). Let $f$ be an isomorphism between $P$ and $i(P)$. Then $P$ is algebraic, and in particular up-complete. Since the compact elements of $i(P)$ are precisely the principal ideals of $P$, and since $f$ is an isomorphism, we conclude that $x \in P$ is compact if and only if $f(x)$ is a principal ideal. Now assume there exists a non-compact element in $P$. As directed joins of non-compact elements are never compact, Zorn's Lemma ensures the existence of a maximal non-compact element $x_{0}$. Then $Y=f\left(x_{0}\right)$ is a directed lower set but not a principal ideal. Hence $x_{1}=V Y$ is not compact, and $\downarrow x_{1}$ is a member of $i(P)$ properly containing $Y$. The map

$$
g: P \rightarrow P, \quad x \mapsto f^{-1}(\downarrow x)
$$

is obviously an order embedding, and $x_{0}<g\left(x_{1}\right)$ (since $f\left(x_{0}\right)=Y \subset \downarrow x_{1}=$ $\left.f\left(g\left(x_{1}\right)\right)\right)$. Repeating this procedure, we obtain a sequence of non-compact elements $x_{0}, x_{1}, x_{2} \ldots$ such that $x_{n}<g\left(x_{n+1}\right)$ for all $n=0,1,2, \ldots$ But then

$$
x_{0}<g\left(x_{1}\right)<g^{2}\left(x_{2}\right)<\cdots<g^{n}\left(x_{n}\right) \cdots
$$

is an ascending chain whose join is not compact. This contradicts the maximality of $x_{0}$. Accordingly, every element of $P$ must be compact.

COROLlary 3. If $P$ is an algebraic poset, then $\kappa(P) \cong P$ already implies that $\kappa(P)=P$.

This implication cannot be extended to arbitrary up-complete posets.

EXAMPLE 2. Consider the chain

$$
C=\{x \in \mathbb{R}: 0<x \leqslant 1 / 2\} \cup\{1\}
$$

with its usual order $\leqslant$. Let $C^{m}$ denote the mth lexicographic power of $C$ $(m=1,2, \ldots)$ and form the ordinal sum

$$
P=1+C+C^{2}+C^{3}+\cdots .
$$


Then the dual poset $P^{*}$ is an up-complete chain (which becomes complete by adjoining a least element), and the following assignment defines an isomorphism between $P^{*}$ and $\kappa\left(P^{*}\right)$ :

$$
\begin{aligned}
& 1 \mapsto \frac{1}{2} \in C, \\
& \left(x_{1}, \ldots, x_{m}\right) \mapsto\left(x_{1}, \ldots, x_{m}, \frac{1}{2}\right) \in C^{m+1} \quad \text { for }\left(x_{1}, \ldots, x_{m}\right) \in C^{m} .
\end{aligned}
$$

\section{Sums and products of algebraic posets}

In this section, we collect together a few results concerning the formation of direct sums and direct products of algebraic posets.

First, we notice that the direct sum ("disjoint union") of any family of algebraic posets is again algebraic, but of course, the direct sum of at least two complete (algebraic) lattices is never complete. However, when a greatest and a least element are adjoined to a disjoint union of complete algebraic lattices, a new complete algebraic lattice is obtained in which the greatest element is compact. A straightforward computation shows that direct sums are in fact co-products in the category AP because the natural injections preserve directed joins and compactness.

On the other hand, it is well known that arbitrary products of complete algebraic lattices are algebraic. This result can be extended only in part to algebraic posets, as we shall see below.

Call a poset $P$ uniquely minimized if for every $x \in P$ there exists a unique minimal element $\tilde{x} \leqslant x$. Thus, for example, each poset possessing a least element and every anti-chain is uniquely minimized. More generally, a poset is uniquely minimized if and only if it is a disjoint union of posets each of which has a least element. In this case, the minimal elements are obviously compact.

Proposition 5. A direct product $P$ of posets $P_{j}(j \in J)$ is algebraic if and only if each $P_{j}$ is algebraic and the number of indices $j$ for which $P_{j}$ is not uniquely minimized is finite.

PROOF. Since the projections preserve directed joins and compactness, it is clear that each factor $P_{j}$ of an algebraic product poset $P=\prod_{j \in J} P_{j}$ must be algebraic.

Assume that the set $K=\left\{j \in J: P_{j}\right.$ is not uniquely minimized $\}$ is infinite. Choose $y \in P$ such that for no $j \in K$ is there exactly one minimal element dominated by $y_{j}$. Then we assert that no element $x \leqslant y$ can be compact in $P$ (and in particular, $P$ cannot be algebraic). Indeed, we may choose an element $z \leqslant y$ 
with $x_{j} \nless z_{j}$ for all $j \in K$. Thus

$$
Y=\left\{w \in P: w \leqslant y ;\left\{j \in J: w_{j} \nless z_{j}\right\} \text { finite }\right\}
$$

is a directed lower set in $P$ with $y=\vee Y$ but $x \notin Y$.

On the other hand, assume that each $P_{j}$ is algebraic and that there exists a finite set $K \subseteq J$ such that, for all $j \in J \backslash K$ and all $v \in P_{j}$, there is a unique minimal element $\tilde{v} \leqslant v$. Then the product poset $P=\prod_{j \in J} P_{j}$ is up-complete, and for each $y \in P$, the set

$$
K_{y}=\left\{x \in P: x \leqslant y ; x_{j} \text { compact in } P_{j} \text { for all } j \in J ;\left\{j \in J: x_{j} \neq \tilde{y}_{j}\right\} \text { finite }\right\}
$$

is a directed (non-empty!) set of compact elements in $P$ with $y=V_{K_{y}}$.

Corollary 4. (1) The class of algebraic posets is closed under finite direct products and arbitrary direct sums.

(2) The class of uniquely minimized algebraic posets is closed under arbitrary direct products and direct sums.

(3) The class of algebraic posets with least elements is closed under arbitrary direct products.

It is easy to see that these direct products and sums have the usual categorical properties of products and coproducts with respect to the AP-morphisms. Moreover, the category AP of algebraic posets has not only finite but arbitrary products, since AP is equivalent to the category $P$ of partially ordered sets, where arbitrary direct products are the products in the categorical sense. However, in AP a product with an infinite number of factors may be distinct from the usual direct product. In the general case, one has to take the up-completion $i\left(\Pi_{j \in J} \kappa\left(P_{j}\right)\right)$ as a representative for the categorical product of the algebraic posets $P_{j}(j \in J)$.

From Proposition 5 we infer that the direct product of a family of (non-empty) algebraic $\wedge$-semilattices is algebraic if and only if the number of factors without least element is finite. Moreover, one has the following result (which is shown in the same way as Proposition 5): if $\left(P_{j}: j \in J\right)$ is an infinite family of non-empty posets none of which has a minimal element, then the direct product $P=\prod_{j \in J} P_{j}$ contais no compact elements at all.

ExAmple 3. The chain $C=\{-1,-2,-3, \ldots\}$ of all negative integers is algebraic; moreover, each element of $C$ is compact. Hence the same holds for each finite power $C^{n}$. But no infinite cartesian power $C^{m}$ contains any compact element at all. The $m$-th power of $C$ in the category AP is the up-completion $i\left(C^{m}\right)$. 


\section{Substructures and weak atomicity}

A straightforward computation shows that every Scott-closed subset and every Scott-open subset of an algebraic poset is again an algebraic poset (with respect to the induced order). For example, the Scott-closed subsets of a power set (which is certainly an algebraic complete lattice) are precisely the systems of finite character, and we have already remarked that such systems are special instances of algebraic posets.

It is also well-known that every complete sublattice and, in particular, every interval $[a, b]=\{x \in L: a \leqslant x \leqslant b\}$ of an algebraic complete lattice $L$ is again algebraic, although the compact elements of $[a, b]$ may be completely different from those of $L$. Unfortunately, this relativization property no longer holds for algebraic posets.

\section{EXAMPLE 4.}

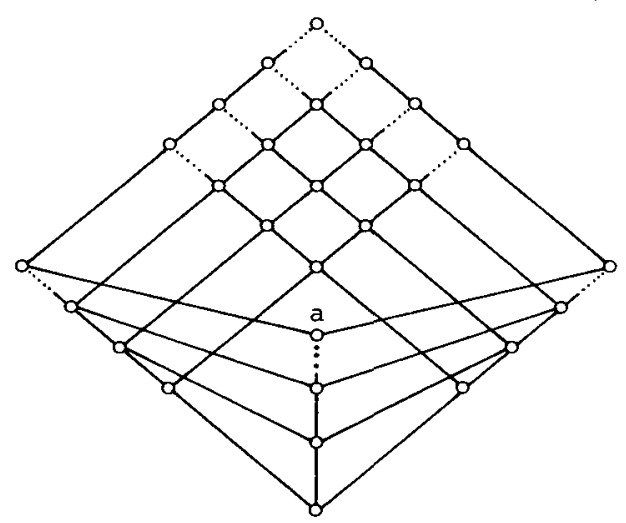

Figure 1

Figure 1 represents an algebraic poset containing an interval $[a, 1]$ which is a complete lattice but certainly not compactly generated. Indeed, the element $a$ is the only compact element of the interval $[a, 1]$ while $a$ is not compact in the entire poset.

A poset $P$ is said to be weakly atomic if every interval $[a, b]$ with $a<b$ contains a jump, i.e. a pair of elements $u<v$ such that $[u, v]=\{u, v\}$. It has been observed by Birkhoff and Frink [3] that every algebraic complete lattice $L$ is weakly atomic. However, the initial proof given in [3] contained a little gap which was closed by K.-H. Diener [6]. A straightforward argument is this: if $a<b$, then there exists a compact element $v$ of $[a, b]$ (which need not be compact in $L$ ) such 
that $a<v$. By Zorn's Lemma, we can find a maximal element $u$ with $a \leqslant u<v$. Thus $[u, v]$ is the required jump. The same argument shows that a poset in which every interval is algebraic must be weakly atomic, but an algebraic poset need not be weakly atomic.

Example 5. For $a, b \in \mathbb{R}$, set $[a, b[=\{x \in \mathbf{R}: a \leqslant x<b\}$, and set $L=$ $\{[0, a]: a \in[0,1[\} \cup\{[0, a[: a \in[0,1]\}$. Then $L$ is a complete chain with respect to inclusion, consisting of all lower sets of the interval [0,1[. Hence $L$ is an algebraic lattice, and the compact elements of $L$ are the intervals $[0, a]$ with $a \in[0,1[$ and the empty interval $\varnothing=[0,0[$.

If $\omega$ denotes the chain of all natural numbers $0,1,2, \ldots$ then $\omega+1=\omega \cup\{\omega\}$ is an algebraic chain whose compact elements are the natural numbers. Consider the set

$$
P=(\omega \times L) \cup\{(\omega,[0, a[): a \in[0,1]\} \subseteq(\omega+1) \times L,
$$

together with the partial order

$$
(m, I) \leqslant(n, J) \Leftrightarrow m \leqslant n, I \subseteq J \text {, and } I=J=[0, a] \text { implies that } m=n .
$$

It is not hard to verify that $P$ is an algebraic poset, the compact elements being the pairs $(n, I)$ with $n \in \omega$, and with $I=\varnothing$ or $I=[0, a]$ for some $a \in[0,1[$. But the interval $[(\omega, \varnothing),(\omega,[0,1[)]=\{(\omega,[0, a[): a \in[0,1]\} \subseteq P$ is isomorphic to the unit interval $[0,1]$ and contains no jump.

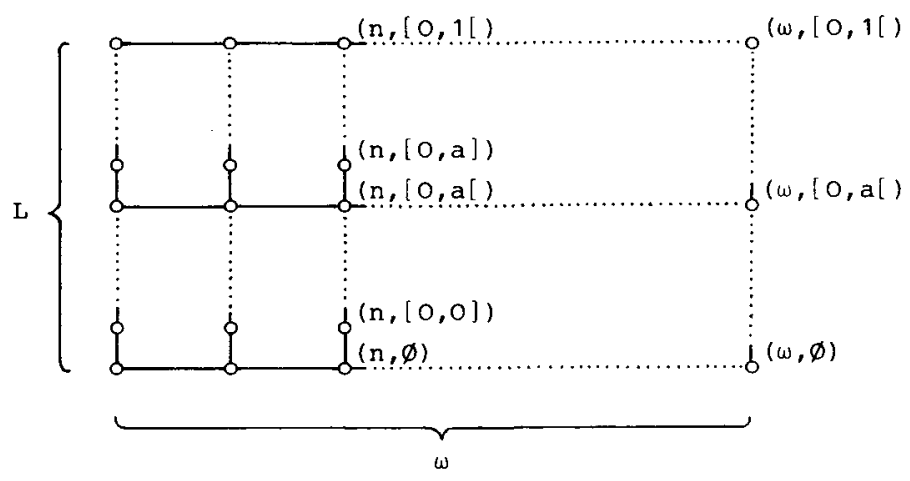

Figure 2

In spite of this counterexample, we can prove a slight generalization of the fact that algebraic complete lattices are weakly atomic. Call a poset $P$ chain-complete or Dedekind complete (cf. Wolk [17]) if every non-empty chain of $P$ has a join and a meet (in other words, if $P$ and its dual $P^{*}$ are up-complete). 
PROPOSITION 6. Every compactly generated chain-complete poset is weakly atomic.

Proof. Let $[a, b]$ be an interval of a compactly generated chain-complete poset $P$ with $a<b$, and let $C$ be a maximal chain in $[a, b]$. Then $C$ must be complete, and joins and meets in $C$ agree with those formed in $P$. Choose a compact element $c$ of $P$ with $c \leqslant b$ but $c \notin a$. Let $v=\wedge\{z \in C: c \leqslant z\}$. Then $c \leqslant v \in C$ (!) and $a<v$. Furthermore, $D=\{z \in C: z<v\}$ is a chain with $c \notin z$ for all $z \in D$, whence, by compactness of $c, c \star u=V D$, and so $u<v$. Thus [ $u, v]$ is a jump in $[a, b]$, as desired.

Notice that the proofs of Diener [6] and Crawley and Dilworth $[5,2.2]$ for the weak atomicity of algebraic lattices involve the existence of certain finite (undirected) joins and do not work in the present more general setting.

\section{Meet-decompositions in compactly generated posets}

The famous decomposition theorem for algebraic lattices due to Birkhoff and Frink [3] extends without any alteration to compactly generated posets.

Proposition 7. Every element of a compactly generated poset is a meet of completely meet-irreducible elements.

By a completely meet-irreducible ( $\Lambda$-irreducible) element of a poset $P$ we mean an element $q$ which cannot be represented as the meet (greatest lower bound) of a set $Y \subseteq P$ unless $q \in Y$. Notice that a maximal element of a poset is $\Lambda$-irreducible if and only if it is not the greatest element. In view of Proposition 2, the above decomposition theorem is only a modification of the following version.

Proposition 8. Let $\mathfrak{X}$ be an inductive standard extension of a poset $Q$. Then each member of $\mathfrak{X}$ is an intersection of $\wedge$-irreducible members of $\mathfrak{X}$.

In other words, given $X \in \mathfrak{X}$ and $x \in Q \backslash X$ we find a $\Lambda$-irreducible $Y \in \mathfrak{X}$ with $X \subseteq Y$ and $x \notin Y$.

This is an immediate consequence of Zorn's Lemma; indeed, a maximal $Y \in \mathfrak{X}$ with $X \subseteq Y$ and $x \notin Y$ must be $\Lambda$-irreducible because every $Z \in \mathfrak{X}$ properly containing $Y$ also contains the element $x$.

We conclude this note with a few applications.

Let $\mathfrak{V}$ be an inductive system which is closed under intersections of arbitrary non-empty subsystems (for example, each system of finite character has this 
property). Then $\mathfrak{V}$ is an algebraic poset. In fact, $\mathfrak{X}=\mathfrak{Y} \cup\{\cup \mathfrak{Y}\}$ is even an algebraic closure system whose greatest element is compact iff $\mathfrak{X} \neq \mathfrak{V}$. The compact members of $\mathfrak{X}$ are the finitely generated ones, i.e. the sets $\langle Z\rangle=$ $\cap\{Y \in \mathfrak{X}: Z \subseteq Y\}$ with finite $Z \subseteq \cup \mathfrak{Y}$.

EXAMPLE 6. Let $\mathscr{Q}_{0}(X)$ denote the set of all partial orders definable on a set $X$ (considered as subsets of the cartesian product $X \times X)$. Obviously $\mathscr{Q}_{0}(X)$ is closed under directed unions and under intersections of non-empty subsystems. However, $\mathscr{Q}_{0}(X)$ is neither a closure system (missing a greatest element) nor a system of finite character $(|X|>1)$. Nevertheless, $\mathscr{Q}_{0}(X)$ is an algebraic poset, and consequently every partial order is the intersection of $\Lambda$-irreducible ones. But it is easy to see that the $\Lambda$-irreducible members of $\mathscr{Q}_{0}(X)$ are precisely the maximal ones, in other words, the linear orders on $X$. Thus the well-known fact that every partial order is an intersection of linear orders may be viewed as a special case of Proposition 7.

EXAMPLE 7. Since the system of all chains of a fixed poset $P$ is of finite character, we infer that every chain of $P$ is an intersection of $\Lambda$-irreducible ones. If $P$ itself is a chain, then the $\Lambda$-irreducible subchains are precisely the co-atoms of the power set $\mathfrak{B}(P)$. But in general, a $\Lambda$-irreducible subchain of a non-linearly ordered poset $P$ need not be maximal. In fact, one can check easily that a chain $C \subseteq P$ is $\Lambda$-irreducible if and only if it is maximal, or there exists a unique element $x \in P \backslash C$ such that $C \cup\{x\}$ is a maximal chain.

EXAMPLE 8. Consider a fixed vector space $V$ with $|V|>2$. We know that the system of linearly independent subsets of $V$ is of finite character, and hence is an algebraic poset. Thus every linearly independent subset of $V$ is a meet (that is, an intersection) of $\Lambda$-irreducible ones. But a non-maximal linearly independent subset cannot be $\Lambda$-irreducible. So we conclude that every linearly independent subset of a vector space $V$ is an intersection of bases (unless $|V|=2$ ).

EXAMPLE 9. Let $\mathfrak{B}$ denote the system of all well-ordered subsets of a fixed poset $P . \mathfrak{B}$ is partially ordered by "propagation":

$$
W_{1} \preccurlyeq W_{2} \Leftrightarrow W_{1} \text { is a lower set of } W_{2} \text {. }
$$

The compact elements of $\mathfrak{B}$ are those which are not isomorphic to limit ordinals. $\mathfrak{B}$ is an algebraic poset and closed under non-empty intersections. Hence every well-ordered subset of $P$ is an intersection of $\Lambda$-irreducible ones (with respect to 
propagation). Similarly to Example 7, a non-maximal $W \in \mathfrak{B}$ is $\Lambda$-irreducible if and only if $W$ has a unique upper bound. Furthermore, the following four statements are equivalent.

(a) $P$ satisfies the descending chain condition.

(b) Every chain of $P$ is well ordered.

(c) $\mathfrak{B}$ is of finite character.

(d) $\mathfrak{B}$ is inductive.

Each of the previous examples becomes an algebraic complete lattice by adjoining a greatest element. In contrast, our final example concerns an algebraic poset which is not even a $\vee$ - or a $\wedge$-semilattice.

EXAMple 10. For $x \in \mathbb{R}^{n}$ and $\varepsilon \geqslant 0$, let

$$
D_{x, \varepsilon}:=\left\{y \in \mathbb{R}^{n}:\|x-y\| \leqslant \varepsilon\right\}
$$

denote the closed ball with center $x$ and radius $\varepsilon$ (where \|\| denotes the usual Euclidean norm). In particular,

$$
E=D_{0,1}=\left\{y \in \mathbb{R}^{n}:\|y\| \leqslant 1\right\}
$$

denotes the unit ball. The system

$$
\mathcal{F}:=\left\{D_{x, \varepsilon}: x \in E, \varepsilon \geqslant 0, D_{x, \varepsilon} \subseteq E\right\}
$$

of all closed balls contained in the unit ball is up-complete but not inductive. Furthermore, $\subseteq$ neither contains compact elements (the points are not compact in (5) nor $\Lambda$-irreducible elements. Now let

$$
\mathfrak{U}:=\{\stackrel{D}{D}: D \in \mathbb{E}\} \backslash\{\varnothing\}
$$

denote the system of all open balls contained in $E . \mathfrak{A}$ is inductive, but again $\mathfrak{A}$ has neither compact nor $\Lambda$-irreducible elements. However, it can be shown that $\mathfrak{A}$ is a continuous poset while $\mathfrak{E}$ is not. Finally, define

$$
\begin{aligned}
& \mathfrak{D}:=\mathfrak{A} \cup \mathfrak{B} \cup \mathbb{\mathfrak { C }}, \text { where } \\
& \mathfrak{B}:=\{\dot{D} \cup\{y\}: D \in \mathbb{E} ; y \in D \backslash \dot{D}\}
\end{aligned}
$$

consists of all balls contained in $E$ which have exactly one boundary point.

For $n=1, \mathscr{D}$ is simply the system of all (non-empty) convex subsets of the interval $[-1,1] ; \varnothing \cup\{\varnothing\}$ is an inductive closure system and therefore an algebraic complete lattice. The $\Lambda$-irreducible members of $D$ are the half-open intervals $] a, 1](a \in[-1,1)$ and $[-1, b[(b \in]-1,1])$.

A completely different situation holds for $n>1$. In this case, the following facts are easily checked.

(1) None of the systems $\mathfrak{A}, \mathfrak{B}, \mathfrak{C}$ or $\mathfrak{D}$ is a $\dot{V}$ - or a $\wedge$-semilattice.

(2) $D$ is the least inductive system containing $\mathbb{C}$. 
(3) $\mathbb{E}$ is the set of all compact elements of $\mathfrak{D}$.

(4) $\mathfrak{D}$ is an algebraic poset.

(5) $\mathfrak{B} \backslash\{\{x\}: x \in E\}$ is the set of all $\Lambda$-irreducible elements of $\mathfrak{D}$.

(6) Each element of $\mathfrak{D}$ is an intersection of $\Lambda$-irreducible ones.

\section{References}

[1] P. S. Alexandroff, 'Diskrete Räume', Mat. Sb. (N.S.) 2 (1937), 501-518.

[2] B. Banaschewski, 'Hüllensysteme und Erweiterung von Quasi-Ordnungen', Z. Math. Logik Grundlagen Math. 2 (1956), 369-377.

[3] G. Birkhoff and O. Frink, 'Representation of lattices by sets', Trans. Amer. Math. Soc. 64 (1948), 299-316.

[4] P. M. Cohn, Universal algebra (Harper and Row, New York, 1965).

[5] P. Crawley and R. P. Dilworth, Algebraic theory of lattices (Prentice Hall, Inc., Englewood Cliffs, N. J., 1973).

[6] K.-H. Diener, 'Über zwei Birkhoff-Frinksche Struktursätze der allgemeinen Algebra', Arch. Math. 7 (1956), 339-346.

[7] M. Erné, 'Scott convergence and Scott topology in partially ordered sets II', (in Continuous lattices, Lecture Notes in Math. 871, Springer, Berlin, Heidelberg, New York, 1981).

[8] M. Erné, 'On the existence of decompositions in lattices', Algebra Universalis, to appear.

[9] M. Erné, 'Adjunctions and standard constructions for partially ordered sets' (Contributions to general algebra II, Proc. Klagenfurt Conference 1982, to appear).

[10] G. Gierz, K. H. Hofmann, K. Keimel, J. D. Lawson, M. Mislove and D. S. Scott, $A$ compendium of continuous lattices (Springer, Berlin, Heidelberg, New York, 1980).

[11] D. Higgs, 'Lattices isomorphic to their ideal lattices', Algebra Universalis 1 (1971), 71-72.

[12] R.-E. Hoffmann, 'Sobrification of partially ordered sets', Semigroup Forum 17 (1979), 123-138.

[13] R.-E. Hoffmann, 'Continuous posets and adjoint sequences', Semigroup Forum 18 (1979), 173-188.

[14] R.-E. Hoffmann, 'Continuous posets, prime spectra of completely distributive complete lattices, and Hausdorff compactifications' (in Continuous lattices, Lecture Notes in Math. 871, Springer, Berlin, Heidelberg, New York, 1981).

[15] J. D. Lawson, 'The duality of continuous posets', Houston J. Math. 5 (1979), 357-386.

[16] J. Mayer-Kalkschmidt and E. Steiner, 'Some theorems in set theory and applications in the ideal theory of partially ordered sets', Duke Math. J. 31 (1964), 287-390.

[17] E. S. Wolk, 'Dedekind completeness and a fixed point theorem', Canad. J. Math. 9 (1957), 400-405.

[18] J. B. Wright, E. G. Wagner and J. W. Thatcher, 'A uniform approach to inductive posets and inductive closure', Theoret. Comput. Sci. 7 (1978), 57-77.

Institut für Mathematik

Universität Hannover

D-3000 Hannover 1

Federal Republic of Germany 